\section{Experiencias, barreras y facilitadores en la implementación de intervenciones de control del Aedes aegypti en América Latina y Caribe: estudio cualitativo}

\section{Experiences, barriers and facilitators to the implementation of interventions for controlling} Aedes aegypti in Latin America and the Caribbean: a qualitative study

\section{Experiências, barreiras e facilitadores na implementação de intervenções de controle do Aedes aegypti na América Latina e no Caribe: estudo qualitativo}

\section{Resumen}

El objetivo fue describir experiencias, barreras y facilitadores en la implementación de intervenciones de control del Aedes aegypti en América Latina y Caribe. Fue realizado un abordaje cualitativo con entrevistas en profundidad semiestructuradas a expertos en implementación de programas (19 participantes de nueve países). Se utilizó un software para la codificar los datos y se confeccionaron matrices para su comparación. En base a los hallazgos desarrollamos una representación gráfica de dimensiones teóricas que agrupan las barreras y facilitadores para la implementación de intervenciones. A nivel global, el ambiente natural y construido contribuye a la reproducción del mosquito. En el sistema de salud, la falta de priorización del problema y la escasez de recursos materiales y humanos representan los obstáculos más importantes. Se necesita que otros sectores diferentes al de salud se responsabilicen de las acciones para mejorar los determinantes sociales de la salud. Existen barreras transversales relacionadas con la gobernanza, como la descoordinación entre los niveles centrales y locales, falta de continuidad de las intervenciones y de los grupos técnicos. La comunidad enfrenta problemas como la falta de información, pobreza o resistencias a recomendaciones. La opinión pública puede tener un impacto positivo o negativo, influyendo indirectamente en las decisiones politicas. Este estudio propone un marco de dimensiones de análisis construido desde los hallazgos; describe factores influyentes en la implementación de politicas que pueden orientar las acciones futuras desde un enfoque integrado.

Control de Vectores; Aedes; Análisis Cualitativo
Elena Tapia-López 1,2

Ariel Bardach 1,2,3

Agustín Ciapponi 1,2,3,4

Andrea Alcaraz 1,2

Herney Andrés García-Perdomo 5

Silvina Ruvinsky 6

María Belizán 1,2

doi: 10.1590/0102-311X00092618

\author{
Correspondencia \\ A. Ciapponi \\ Instituto de Efectividad Clínica y Sanitaria. \\ Dr. Emilio Ravignani 2024, Buenos Aires/CABA - \\ C1414CPV, Argentina. \\ aciapponi@iecs.org.ar \\ 1 Instituto de Efectividad Clínica y Sanitaria, \\ Buenos Aires, Argentina. \\ 2 Centro de Investigación de Epidemiología y Salud Pública, \\ Buenos Aires, Argentina. \\ 3 Consejo Nacional de Investigaciones Científicas y Técnicas, \\ Buenos Aires, Argentina. \\ 4 Centro Cochrane Argentino, Buenos Aires, Argentina. \\ 5 Universidad del Valle, Cali, Colombia. \\ ${ }^{6}$ Hospital Dr. Juan P. Garrahan, Buenos Aires, Colombia.
}




\section{Fundamentación}

El Aedes aegypti es el mosquito transmisor del dengue, chikungunya, Zika y fiebre amarilla. Está presente en casi todos los países de América, excepto Canadá y Chile, tanto en ámbitos urbanos como selváticos 1 .

Las enfermedades transmitidas por Ae. aegypti imponen una pesada carga sanitaria para la región. Ya en el 2015, se confirmó tanto un aumento en la prevalencia de microcefalia, insuficiencia placentaria, retraso en el crecimiento y muerte fetal, asociados a la infección del virus del Zika durante el embarazo, como también se relacionó la infección por Zika con un incremento en los casos de síndrome de Guillain-Barré. Esto determinó que el 1o de febrero del 2016 la Organización Mundial de la Salud (OMS) declarase una emergencia de salud pública de importancia internacional y recomendase intensificar las actividades de vigilancia e investigación relacionadas con este virus 2.

Por otro lado, aproximadamente 2.500 millones de personas viven en zonas donde el dengue es endémico y se reportan de 50 a 100 millones de nuevos casos anualmente 3 . La incidencia mundial de dengue y dengue grave ha aumentado en los últimos 40 años, con una distribución en expansión entre los países. Debido al gran número de casos, se la considera una de las enfermedades virales más importantes transmitidas por artrópodos 4,5 .

Los macrodeterminantes más importantes responsables del desarrollo de la enfermedad son la falta de programas eficaces de control de vectores, el aumento de la densidad de población, las malas condiciones sanitarias en las zonas urbanas, y el deterioro de los sistemas de salud públicos, junto con factores ambientales (niveles de precipitaciones y temperaturas medias) 6,7.

La OMS promueve un enfoque estratégico, conocido como Manejo Integrado de Vectores, definido como el proceso racional de toma de decisiones para optimizar el uso de recursos en el control de vectores, con el objetivo de mejorar la eficacia, costo-efectividad, congruencia ecológica y la sostenibilidad en el tiempo. Las actividades principales en este control se centran en los métodos para el control de vectores, asociados a gestión ambiental, control químico y control biológico, a través de actividades como uso de insecticidas, reducción de reservorios, educación para la salud y comunicación social, vigilancia epidemiológica y vigilancia entomológica 8 .

El control del mosquito Ae. aegypti constituye una de las principales medidas de prevención, aunque lograr ese objetivo representa un desafío para los países en desarrollo. Para abordar la complejidad de este problema sanitario llevamos a cabo tres abordajes complementarios. Los resultados de la investigación que aquí se presenta pueden ser leídos en conjunto con los dos restantes. En primer lugar, se realizó una revisión sistemática para analizar la efectividad y costo-efectividad de las distintas intervenciones; se identificó evidencia sobre seis tipos de intervenciones: cortinas y tules impregnados con insecticidas; educación para la salud y participación comunitaria; uso de insecticidas domiciliarios; manejo de reservorios; ovitrampas-larvitrampas; y vigilancia epidemiológica como parte de un programa de control 9 . La revisión concluyó que ninguna de las intervenciones detectadas tiene alta calidad de la evidencia. Sin embargo, a pesar de tener menor grado de evidencia, algunas de estas intervenciones podrían ser efectivas en reducir los índices entomológicos de densidad de $A e$. aegypti (índices de Breteau, de pupas por persona y de hogares, y densidad de mosquitos adultos) o la incidencia y letalidad de enfermedades transmitidas por ese vector.

En segundo lugar, realizamos una investigación cualitativa, cuyos hallazgos se presentan en este manuscrito. Con toda esta valiosa información se realizó un diálogo de decisores (policy dialogue), focalizado en la priorización de intervenciones para el control del mosquito Ae. aegypti y de líneas de investigación 10. Mediante el estudio cualitativo se propuso explorar las experiencias del control de Ae. aegypti en los países de América Latina y Caribe. Se exploraron los factores que obstaculizan o facilitan la implementación de intervenciones desde la perspectiva de los responsables de programas y referentes nacionales. 


\section{Metodología}

\section{Diseño del estudio}

Se realizó una investigación inductiva consistente en la teoría fundamentada ${ }^{11}$ y ejecutada de acuerdo a las pautas establecidas para la investigación cualitativa 12,13,14. Se incluyeron entrevistas en profundidad semiestructuradas con expertos.

El estudio fue realizado entre julio de 2016 y julio de 2017. El protocolo de investigación fue aprobado por el Comité de Ética de la Universidad del Valle, Cali, Colombia.

\section{Participantes y muestra}

Mediante un muestreo teórico intencional se seleccionaron actores clave en nueve países de América Latina y Caribe que presentaran carga de enfermedad y poseyeran programas en marcha: Argentina, Brasil, Colombia, Cuba, Honduras, Panamá, Paraguay, Perú, Puerto Rico y a expertos regionales de la OMS y la Organización Panamericana de la Salud (OPS).

Se buscaron informantes clave que tuvieran experiencia y conocimiento sobre la implementación de programas nacionales o regionales como ejecutores de programas, técnicos e investigadores. Durante la recolección de datos se decidió también entrevistar a representantes de organizaciones no gubernamentales (ONGs) que trabajan en prevención.

\section{Recolección de datos}

Se invitaron a 28 expertos a través de correos electrónicos durante los meses de agosto y setiembre del 2016. Las entrevistas fueron realizadas telefónicamente o por Skype (https://www.skype.com). Todos los entrevistados dieron su consentimiento verbal antes de comenzar. Las entrevistas tuvieron una duración de entre 30 y 60 minutos y fueron grabadas en audio y transcritas en verbatims en preparación para el análisis.

La guía de preguntas se orientó a conocer las experiencias de acciones implementadas durante los últimos cinco años, desde la fecha de la entrevista. Se indagó sobre los programas o intervenciones implementadas en los países, barreras y facilitadores en su aplicación, brechas en la investigación, y opiniones sobre acciones prioritarias.

\section{Análisis de datos}

Las transcripciones fueron introducidas en el programa de análisis de datos ATLAS.ti versión 8.1 (http://atlasti.com/) y fueron codificadas de acuerdo a un manual de códigos, desarrollado en base a las dimensiones incluidas en las preguntas del cuestionario y suplementadas por temas emergentes. Se realizó un análisis temático por cada país. Se desarrolló una matriz para facilitar la comparación transversal de los datos, reteniendo el contexto de los entrevistados. Finalmente, los datos fueron interpretados.

Como parte del análisis, se seleccionaron fragmentos textuales de las opiniones de los participantes (presentados en el Cuadro 1), sin revelar su identidad para preservar la confidencialidad.

Dada la heterogeneidad en las condiciones sociales y políticas de la región, en la descripción de los hallazgos se recoge la totalidad de las opiniones encontradas. El propósito de ser inclusivos es brindar a los decisores, independientemente del país o región a la que pertenezca, un marco útil que pueda adaptarse a su propia situación. 


\section{Cuadro 1}

Hallazgos de barreras por dimensiones de análisis.

\begin{tabular}{|c|c|c|}
\hline $\begin{array}{l}\text { DIMENSIONES DE } \\
\text { ANÁLISIS }\end{array}$ & BARRERAS & DECLARACIONES LITERALES \\
\hline \multirow[t]{3}{*}{ Ambiente físico } & $\begin{array}{l}\text { - Impredecibilidad de la } \\
\text { aparición del Ae. aegypti }\end{array}$ & $\begin{array}{c}\text { "Yo puedo hacer las mejores acciones posibles, pero yo no sé si una baja ocurrencia } \\
\text { de casos está relacionada con el resultado de mis acciones o tiene que ver con el ciclo } \\
\text { natural del virus que hace que ese año no ocurriera como el año pasado" }\end{array}$ \\
\hline & $\begin{array}{l}\text { - Condiciones climáticas } \\
\text { favorables a la permanencia } \\
\text { del Ae. aegypti }\end{array}$ & $\begin{array}{c}\text { "El cambio climático está facilitando que aparezcan estos vectores durante todo el año y } \\
\text { no sólo en estaciones" }\end{array}$ \\
\hline & $\begin{array}{l}\text { - Condiciones de } \\
\text { reproducción del vector }\end{array}$ & \\
\hline \multirow[t]{8}{*}{ Sistema de salud } & $\begin{array}{l}\text { - Falta de priorización del } \\
\text { problema sanitario }\end{array}$ & $\begin{array}{c}\text { "En [la capital del país] se da un buen ejemplo (...) de que la variable que determina el } \\
\text { éxito o el fracaso es la decisión política, porque [en la capital] no podemos aducir que el } \\
\text { tema pasa por una cuestión presupuestaria, es pura decisión política" }\end{array}$ \\
\hline & $\begin{array}{l}\text { - Falta de continuidad de los } \\
\text { programas o iniciativas }\end{array}$ & $\begin{array}{c}\text { "El problema es que no hay continuidad y contigüidad de las políticas, no existe } \\
\text { esa cultura, y por eso se fracasa en el control epidemiológico"; "Los programas de } \\
\text { erradicación del Aedes aegypti han tenido un proceso de discontinuidad en los países de } \\
\text { la región. No han logrado ser sostenibles" }\end{array}$ \\
\hline & $\begin{array}{l}\text { - Falta de acciones } \\
\text { preventivas }\end{array}$ & $\begin{array}{l}\text { "Los programas no son sostenibles para acciones de prevención y control, muchas veces } \\
\text { solamente se responde a situaciones de brotes o de emergencia"; "No se está haciendo } \\
\text { prevención, se está enfocando la acción en el nivel control" }\end{array}$ \\
\hline & - Escasa financiación & $\begin{array}{c}\text { "A pesar de que existe un financiamiento por parte del gobierno central, muchas veces } \\
\text { por la propia descentralización que existe, las regiones no hacen uso adecuado del } \\
\text { presupuesto brindado, entonces el problema de dengue es la intersectorialidad" }\end{array}$ \\
\hline & - Falta de recursos apropiados & $\begin{array}{c}\text { "Las familias se quejaban de que el rociado del insecticida no duraba mucho tiempo, } \\
\text { entonces luego de evaluar donde estaba el error se percataron que las máquinas } \\
\text { utilizadas para el rociado no habían tenido un adecuado mantenimiento, entonces } \\
\text { las gotas que salían eran grandes y caían rápidamente al suelo en lugar de quedarse } \\
\text { vaporizado en el ambiente" }\end{array}$ \\
\hline & - Falta de personal capacitado & $\begin{array}{l}\text { "Otra barrera es que la medicina no cuenta con la calidad de recursos humanos } \\
\text { requeridos, no cuenta con recursos humanos capacitados para la dirección y evaluación } \\
\text { técnica de las intervenciones"; "Es necesario invertir en formación de personal para así } \\
\text { tener equipos multidisciplinarios que puedan apoyar iniciativas y poner en práctica lo } \\
\text { que se acuerda"; "El equipo que se capacita no suele quedarse o permanecer entonces } \\
\text { lo que se trata de hacer es buscar estrategias para que el personal de salud capacitado } \\
\text { permanezca estable en una región, pero este es un problema de políticas y con } \\
\text { transferencias de personal" }\end{array}$ \\
\hline & $\begin{array}{l}\text { - Dificultades para la } \\
\text { evaluación del impacto de las } \\
\text { intervenciones }\end{array}$ & $\begin{array}{c}\text { "A pesar de la implementación de programas adecuados en algunas provincias, } \\
\text { no se logró la evaluación de forma adecuada ni de forma sostenida"; "No se hace } \\
\text { vigilancia entomológica usualmente porque no es prioridad de los ministerio nacional } \\
\text { o provinciales, no se asignan recursos para la prevención. Se actúa ante casos } \\
\text { confirmados" }\end{array}$ \\
\hline & $\begin{array}{l}\text { - Falta de estrategias } \\
\text { de comunicación con la } \\
\text { población }\end{array}$ & $\begin{array}{c}\text { "...hay inconsistencia en la comunicación, es decir como ejemplo, se habla del } \\
\text { descacharrado, pero no se comunica qué significa y el trabajo que se tiene que llevar } \\
\text { a cabo y las comunidades han asumido que descacharrado es retirar la basura } \\
\text { acumulada" }\end{array}$ \\
\hline
\end{tabular}

(continúa) 


\begin{tabular}{|c|c|c|}
\hline $\begin{array}{l}\text { DIMENSIONES DE } \\
\text { ANÁLISIS }\end{array}$ & BARRERAS & DECLARACIONES LITERALES \\
\hline $\begin{array}{l}\text { Otros sectores } \\
\text { diferentes a la salud }\end{array}$ & $\begin{array}{l}\text { - Falta de priorización } \\
\text { o compromiso con } \\
\text { determinantes de la salud } \\
\text { - Falta de partidas } \\
\text { presupuestarias }\end{array}$ & $\begin{array}{c}\text { "Un facilitador de la implementación sería la participación de otros sectores diferentes } \\
\text { al ministerio de salud, pero eso está en trabajo"; "Para que puedan implementarse la } \\
\text { estrategia en los países debe haber decisión política y financiamiento, pero no sólo del } \\
\text { sistema de salud, también de otros sectores"; "El rol de las ONGs es muy importante en } \\
\text { cuanto a la comunicación a la población. El accionar de las ONGs se da en territorios } \\
\text { pequeños, no a nivel nacional" }\end{array}$ \\
\hline \multirow[t]{5}{*}{ Gobernanza } & $\begin{array}{l}\text { - Desarticulación entre nivel } \\
\text { nacional y niveles locales }\end{array}$ & $\begin{array}{l}\text { "Debería haber un mayor participación y estructuras de nivel municipal, porque el control } \\
\text { de Aedes requiere una tarea diaria y es insuficiente lo que la Nación puede aportar, y } \\
\text { además es materialmente imposible estar presente en todos los distritos"; "Los planes } \\
\text { son implementados según las provincias o localidades, en unas funciona mejor que en } \\
\text { otros, la eficiencia varía de acuerdo a las capacidades de gestión y a los recursos de cada } \\
\text { jurisdicción tiene" }\end{array}$ \\
\hline & $\begin{array}{l}\text { - Falta de continuidad } \\
\text { y contigüidad de las } \\
\text { intervenciones }\end{array}$ & $\begin{array}{c}\text { "Contratación por etapas o cortos periodos, hay mucho movimiento de personal } \\
\text { encargado de los programas, generando poco compromiso con el trabajo. Lineamientos } \\
\text { inestables o poco reales a las condiciones de los departamentos y municipios, no están } \\
\text { adaptados a las necesidades y condiciones de ellos" }\end{array}$ \\
\hline & $\begin{array}{l}\text { - Cambio de autoridades/ } \\
\text { gobierno afecta la continuidad } \\
\text { de las políticas }\end{array}$ & "El cambio de gestión que significó un retroceso importantísimo en las acciones previstas" \\
\hline & $\begin{array}{l}\text { - Falta de estabilidad grupos } \\
\text { técnicos a nivel de los } \\
\text { ministerios }\end{array}$ & $\begin{array}{l}\text { "Falta una política de estabilizar los grupos técnicos (...) el tema de darle continuidad a } \\
\qquad \text { los procesos, que no cambien" }\end{array}$ \\
\hline & $\begin{array}{l}\text { - Decisiones de políticos } \\
\text { por encima de las } \\
\text { recomendaciones de los } \\
\text { técnicos }\end{array}$ & $\begin{array}{l}\text { "El político no tiene que tomar decisiones técnicas, el político no le tiene que decir al } \\
\text { técnico cómo controlar el vector, para eso está el técnico, el político lo que tiene que } \\
\text { hacer es generar las condiciones necesarias para que el técnico pueda hacer su trabajo, } \\
\text { esa es la función, en [este país] está todo revertido, el político se cree en condiciones de } \\
\text { poder tomar decisiones y yo creo que es un error cultural" }\end{array}$ \\
\hline \multirow[t]{2}{*}{ Comunidad } & - Pobreza & $\begin{array}{c}\text { "Esas poblaciones, esos grupos, dentro de su entorno cotidiano no tienen percepción } \\
\text { del riesgo de dengue, porque sus necesidades básicas insatisfechas son mucho más } \\
\text { prioritarias para ellos que prevenir el dengue" }\end{array}$ \\
\hline & $\begin{array}{l}\text { - Falta de información } \\
\text { - Falta de sensibilización } \\
\text { - Resistencias a las } \\
\text { intervenciones }\end{array}$ & $\begin{array}{l}\text { "Se resalta el cambio de comportamiento, es decir que la persona sepa que el problema } \\
\text { de transmisión es responsabilidad de él mismo porque en su vivienda crea condiciones } \\
\text { para el desarrollo del vector. Este cambio de comportamiento se viene trabajando desde } \\
\text { el sector educación, pero muchas veces en casa no se refuerza este concepto"; "Aunque la } \\
\text { gente tiene conocimiento de las intervenciones preventivas, se ha fracasado en lograr la } \\
\text { adherencia a las mismas" }\end{array}$ \\
\hline \multirow[t]{2}{*}{ Opinión pública } & $\begin{array}{l}\text { - Presión de los medios de } \\
\text { comunicación sobre políticas } \\
\text { públicas }\end{array}$ & $\begin{array}{c}\text { "La sociedad y los decisores reaccionan ante casos o ante alguna aparición en los medios } \\
\text { que reflejen la aparición de nuevos casos" }\end{array}$ \\
\hline & $\begin{array}{l}\text { - Información difundida } \\
\text { incorrecta cuando no es parte } \\
\text { de campañas formales }\end{array}$ & $\begin{array}{l}\text { "Hay una manipulación de las noticias con un carácter político, la idea sería que alguna } \\
\text { vez podamos no estar dependiendo solamente de los medios y que sean los Estados } \\
\text { los que tomen la delantera de cómo y cuándo informar. Hay que estar informando con } \\
\text { veracidad a la población y sobre todo dando pautas claras, porque la comunidad va a } \\
\text { acompañar (...) la comunidad organizada necesita de alguna manera esas directrices y } \\
\text { para eso están los diferentes secretarios de salud, medio ambiente, control de vectores } \\
\text { que tienen que dar pautas claras, que tienen que utilizar los medios y ser en definitiva } \\
\text { canales oficiales los que nos van manejando esa información" }\end{array}$ \\
\hline
\end{tabular}




\section{Resultados}

\section{Características de los entrevistados}

De los 28 invitados, 19 expertos confirmaron su participación. El número de participantes se conformó de la siguiente manera: Colombia, Cuba, Honduras, Panamá, Paraguay, Puerto Rico y Brasil con un entrevistado por país; en Perú tres entrevistados; en Argentina ocho entrevistados y, además, se entrevistó a un experto regional. La mediana de edad de los entrevistados fue de 55 años (rango 42-75) con una mediana de experiencia en el control del Ae. aegypti de 16 años (rango 3-25); la mayoría fueron varones $(63,2 \%)$.

\section{Marco analítico de factores que pueden obstaculizar (barreras) o facilitar (facilitadores) la implementación de acciones}

Las barreras y facilitadores a la implementación y escalamiento de intervenciones para la prevención y control del Ae. aegypti, emergentes del relato de los entrevistados, fueron agrupadas en seis dimensiones de análisis. Como marco para el desarrollo utilizamos las dimensiones propuestas por Ghaffar et al. 15 en la Matriz de Estrategias Combinadas e incorporamos otras dimensiones surgidas del análisis y que fueron también contempladas por otros autores 16. Tanto las dimensiones como las barreras y facilitadores identificadas, dentro de cada una de las mismas surgen de los datos de este estudio, consisten en constructos teóricos que facilitan el análisis de la situación y que pueden orientar las acciones a tomar. Reconocemos que América Latina y Caribe es una región heterogénea; incluso en el interior de cada país existen condiciones muy diferentes en cuanto al ambiente; los sistemas de salud, otras instituciones y sus recursos; la gobernanza; las condiciones sociales y económicas de las poblaciones; y la influencia de los medios masivos de comunicación o las redes sociales. Los factores que se describen podrían estar ausentes en alguno de los países o regiones dentro de un mismo país. En el detalle de los hallazgos se describen todos los factores hallados sin tener en cuenta cuantas personas lo mencionan.

La Figura 1 organiza e ilustra las dimensiones de análisis donde se agrupan los factores facilitadores y obstaculizadores. La figura, dimensiones y barreras fueron presentadas durante una reunión de expertos, siendo aceptadas y validadas por los participantes 10.

\section{Barreras y facilitadores para la prevención y control de las enfermedades transmitidas por el Aedes aegypti}

En el Cuadro 1 se agrupan las barreras mencionadas por los entrevistados y cada una se ilustra con un verbatims, es decir, con una frase textual de alguno de los entrevistados.

A continuación, describimos las barreras y facilitadores hallados en cada una de las dimensiones de análisis. Se incluyeron todas las barreras y facilitadores mencionadas por los entrevistados sin diferenciar a qué país pertenecen.

\section{- Ambiente}

Esta dimensión está constituida por el clima, ambiente natural y construido, y en ella se incluyen las barreras ambientales que contribuyen a la reproducción del mosquito 16 .

Los entrevistados remarcan que los casos de infecciones con dengue, Zika y chikungunya no tienen un patrón común todos los años. La ocurrencia no sólo depende de las acciones realizadas para prevenirlo, sino de múltiples factores ambientales. Por lo tanto, la impredecibilidad de la aparición de mosquitos en temporadas (variabilidad esperada de cambios en la epidemia) imposibilita la medición del impacto de las acciones ejecutadas.

Se sostiene que las condiciones climáticas de muchos países de América Latina y Caribe son favorables para la permanencia del Ae. aegypti. En la opinión de algunos entrevistados, el cambio climático está facilitando que aparezcan estos vectores durante todo el año, y no sólo en ciertas estaciones, requiriendo fumigación de adultos durante todo el año. Además, se observa que en algunos países hay 


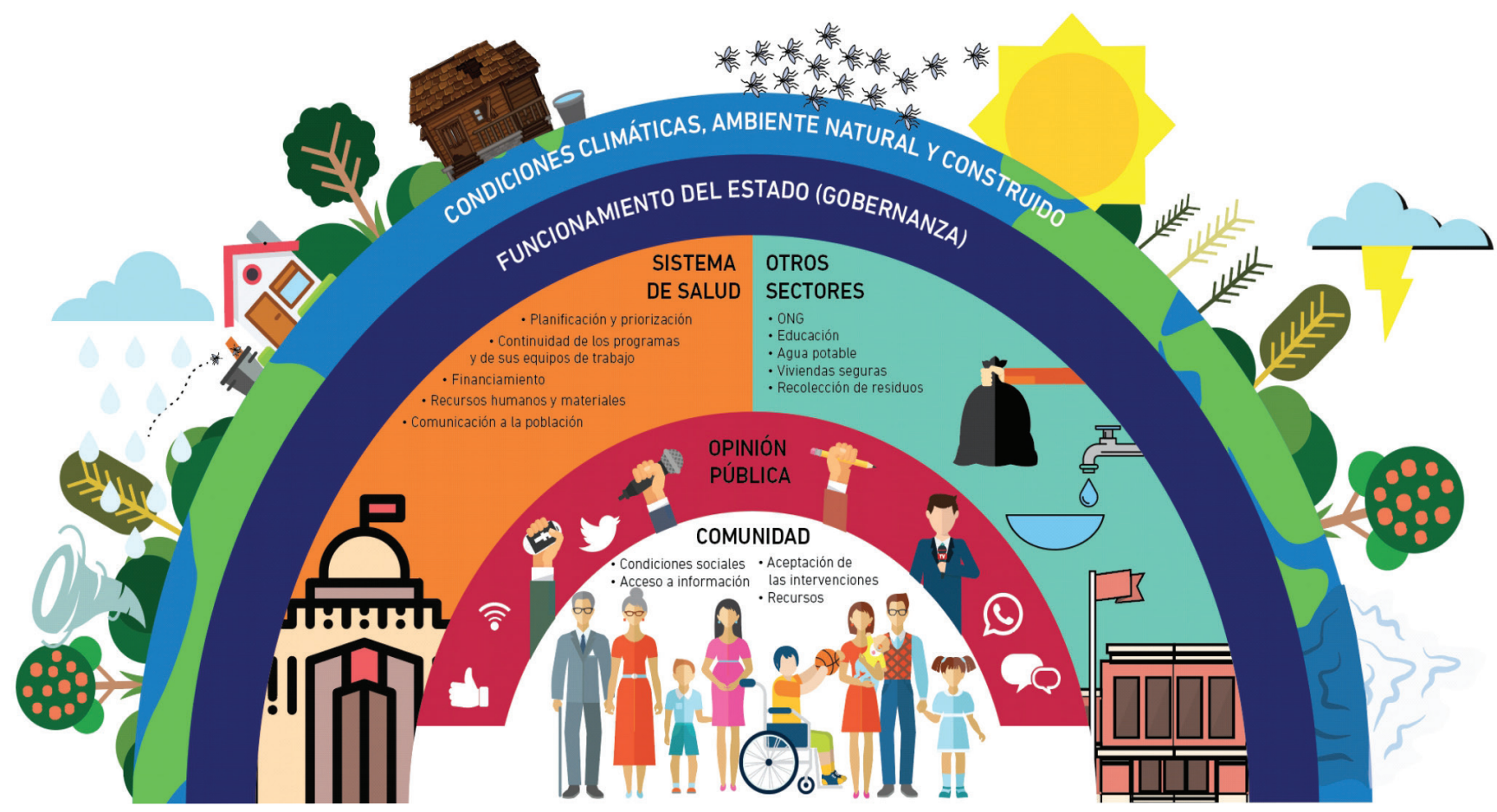

zonas que favorecen a la formación de criaderos y deben tomarse acciones enfocadas a las condiciones particulares de cada lugar; por ejemplo, en zonas con desabastecimiento de agua se dificulta la lucha contra el vector, dado que las comunidades necesitan los reservorios de agua. Este vector puede reproducirse en criaderos urbanos y domicilios.

\section{- Sistemas de salud}

La dimensión de sistema de salud hace referencia al Ministerio de Salud, como el principal cuerpo de las acciones de promoción o prevención y tratamiento de las enfermedades transmitidas por el Ae. aegypti. En esta dimensión se agrupan los factores relacionados con la contribución del sector salud en cuanto a: diseño e implementación de intervenciones, políticas y estructuras que influyen en las condiciones de salud de la población, y también en cuanto a la investigación para proveer métodos y procesos que permitan al sistema de salud reducir las enfermedades transmitidas por el vector 15 .

Muchos entrevistados refirieron que antes de la epidemia de 2009 se enfrentaban a tener que realizar acciones sin una guía concreta que seguir. Sin embargo, la experiencia que se ha tenido ha servido como un facilitador, y hoy creen que hay acuerdo sobre cuáles son las prioridades, y sobre las acciones que se deben implementar. En otras palabras, a día de hoy no sería la principal barrera el no saber cómo actuar, sino que las principales barreras aparecen luego, en la implementación. En varios países se cuenta con programas sostenibles a nivel central.

Una de las mayores barreras en la dimensión del sistema de salud es que el país o el estado, en el caso de los países federales, no priorice este problema sanitario y, en consecuencia, se carece de programas o iniciativas de acciones preventivas sostenidas en el tiempo. Es difícil que se implementen 
programas de vigilancia, ya que no hay recursos asignados para hacerlo. En muchas zonas el dengue es un problema endémico que no ha sido considerado prioritario, aunque la aparición de brotes o la introducción de Zika han abierto las puertas a nuevos recursos técnicos y financieros en estas zonas. Se menciona también que existen intereses políticos de acercarse a la gente para lograr mejorar su imagen o ganar votos, en este sentido tendría menos impacto el brindar información sobre la prevención de las enfermedades transmitidas por este vector que otro tipo de acciones más tangibles como las campañas de vacunación.

Los entrevistados acuerdan que para lograr controlar la transmisión de las enfermedades transmitidas por el Ae. aegypti se necesita una continuidad programática. Sin embargo, en la mayoría de los países de la región se enfrentan con una falta de continuidad de los programas o iniciativas planteadas o recomendadas.

La mayoría de los entrevistados reconoce como barrera la falta de acciones preventivas, las acciones generalmente se implementan en el momento que comienzan a aparecer los casos, de esta forma las intervenciones apuntan a la interrupción de la transmisión. Hacen referencia, por ejemplo, a que las acciones para evitar la aparición de larvas se deben hacer durante todo el año, sin embargo, esto no se realiza en algunos países. En relación al sistema de atención de la salud, se hace referencia a que los centros de atención tradicionalmente están focalizados en la urgencia o en la enfermedad y no en la prevención y promoción.

Muchos de los entrevistados mencionan que la falta de financiamiento puede ser una barrera para la sostenibilidad de acciones. En ocasiones en que las políticas son las apropiadas y las intenciones de acciones están indicadas, se pueden presentar inconvenientes por la falta de recursos a nivel local o la precariedad de los recursos existentes. En muchos sitios no se cuenta con personal capacitado, tanto a niveles de dirección, y evaluación técnica de las intervenciones. En algunos casos se menciona que se hacen esfuerzos en capacitar al personal de salud, pero luego estos no permanecen en sus puestos, perdiendo esa inversión en la formación. Se menciona que en algunos países no se cuenta con entomólogos formados, siendo esto considerado una necesidad imperiosa frente a los cambios de comportamiento de los vectores ante el cambio climático.

Si bien se reconoce que los índices aédicos no son suficientes para la previsión de brotes y epidemias, son datos útiles a considerar y se han hecho esfuerzos por implementar la vigilancia. Algunos de los entrevistados consideran que se ha logrado implementar en los países sistemas de vigilancia y registros de los índices aédicos, pero puede haber fallas en las acciones de vigilancia. Existe una opinión compartida sobre la dificultad de evaluar en qué medida las acciones que se implementan son exitosas o fracasan. Además de las dificultades en evaluar acciones, se puede asumir que en algunas ocasiones puede ser una estrategia de las autoridades no realizar evaluaciones que contradigan las decisiones tomadas. Por ejemplo, tal como señala un entrevistado puede haber resistencias desde los ministerios en revelar los fracasos y por ese motivo se resisten a evaluar o a mostrar los datos.

Algunos países consideran que han logrado implementar acciones de comunicación social adecuadas, sostenibles y efectivas. Según la experiencia de algunos países, se ha trabajado en la comunicación dirigida a los niños en las escuelas, no sólo pensando en que los niños son vulnerables, sino que también son quienes pueden replicar los mensajes y concientizar en los hogares. Sin embargo, otros reportan a falta de estrategias de comunicación a la población o que enfrentan barreras o desafíos en lograr campañas de comunicación efectivas. Consideran que muchas fallan, porque en el diseño de las estrategias de las campañas hay una disociación entre el mensaje construido desde el ministerio y la realidad de las poblaciones.

\section{- Otros sectores diferentes a salud}

Esta dimensión representa los sectores encargados de afrontar ciertos factores o determinantes sociales de la salud, como el acceso al agua potable, viviendas seguras, recolección de residuos, alimentación saludable, educación y entorno están ligados a los resultados en salud. En esta dimensión se agrupan los factores referidos a otros ministerios, departamentos o instituciones, y también a ONGs, que afectan las acciones de prevención y control de las enfermedades transmitidas por el Ae. aegypti 15 .

Según la perspectiva de los entrevistados, para que pueda implementarse la estrategia en los países debe haber decisión política y financiamiento, pero no sólo desde sistema de salud, sino 
también desde otros sectores. Muchos entrevistados resaltan la falta de priorización o compromiso de otros sectores con la atención a los determinantes de la salud. Se necesita que otros sectores (otros ministerios, departamentos u organizaciones de la sociedad civil) con roles importantes, tanto en investigación, como en trabajo comunitario, se responsabilicen también de las acciones. Se destaca que en la mayoría de los países de la región no se asigna presupuesto a otros sectores para acciones relacionadas con la salud.

Es de destacar que, además de los sectores gubernamentales, hay otras organizaciones con amplia experiencia en prevención y promoción de la salud. Llevan a cabo acciones en lugares donde no está presente el estado. En América Latina y Caribe existen ONGs que cumplen un rol importante, tanto en investigación, como en el trabajo con la comunidad. Estas ONGs tienen cercanía con la comunidad y son un nexo valioso para la implementación de acciones tendientes a brindar información y cambiar conductas de riesgo.

\section{- Gobernanza}

Esta dimensión se refiere al funcionamiento del estado en procesos donde los gobernantes son cargos electos; las capacidades de formular e implementar políticas; interacciones y acuerdos entre gobernantes y gobernados, para generar oportunidades y solucionar los problemas de los ciudadanos, y para construir las instituciones y normas necesarias para generar esos cambios 15,17,18,19. Además, incluye a actores no estatales, interconectados por relaciones formales e informales que operan en el proceso de implementación de las políticas. En la dimensión de gobernanza se agrupan las barreras referidas a las estructuras e instituciones a nivel de gobierno central; las decisiones nacionales, provinciales y locales; y su articulación entre estos o con el conjunto de actores no estatales que afectan acciones tendientes a abordar la problemática de las enfermedades transmitidas por el Ae. aegypti. La gobernanza es transversal a las dimensiones de sistema de salud y otros.

Fue mencionado por casi todos los entrevistados que hay una falta de coordinación de políticas de salud con otros ministerios. Esta desarticulación se da tanto por la falta de tradición en la articulación de políticas o por el desinterés de parte de otros sectores en colaborar con acciones sanitarias.

Existe, además, descoordinación entre el nivel nacional y niveles locales. Las políticas impulsadas por gobiernos nacionales muchas veces fracasan en llegar a ser adecuadamente implementadas en las localidades. Muchos de los entrevistados contaron experiencias sobre las diferencias que se dan entre las localidades de un mismo país o provincia, debido a que las acciones propuestas desde los niveles centrales no están coordinadas con las locales. Esta barrera fue mencionada con mayor énfasis en los países federales, donde las provincias o estados tienen diferentes prioridades y cuentan con recursos financieros propios. Se puede dar también que las iniciativas propuestas a nivel nacional no consideren que los efectores locales no tengan los recursos necesarios para implementarlas (tanto de personal capacitado, como de recursos materiales apropiados).

Los problemas de gobernanza dificultan la continuidad en el tiempo de las políticas. Por otro lado, fue remarcada la falta de contigüidad territorial de las políticas, ya sea entre estados en los países federales, como en las zonas limítrofes, conduciendo al fracaso en el control epidemiológico.

Muchos entrevistados remarcaron que ha habido en los últimos años una falta de continuidad programática, debido a cambio de autoridades de gobierno. Consideran que en los períodos de elecciones no se actúa de la forma programada, porque los intereses están más orientados a acciones que tengan mayor impacto en la opinión pública.

Asociado a los problemas relacionados con la continuidad de las políticas se presenta también la falta de estabilidad de grupos técnicos a nivel de los ministerios, cuando cambian las autoridades en muchos casos también se pierde a los expertos que han ganado experiencia. El cambio de las autoridades, como jefes o responsables de programas, implica también el reemplazo del personal que ha sido capacitado por otro sin experiencia. Se considera que hace falta una política de estabilización de grupos técnicos.

Muchos de los países de la región manifestaron que no se ha logrado establecer un programa sostenible o política de estado, basados en las recomendaciones de expertos en el tema. Uno de los motivos es que las decisiones de asignación de recursos se toman en base a la conveniencia política, anteponiéndose a las advertencias de los técnicos expertos. Uno de los entrevistados remarca que los 
programas de salud deben ser políticas de estado, que no se modifiquen ante cambios de gobiernos. Se considera que los técnicos deben tomar decisiones técnicas, porque los políticos no saben de eso.

\section{- Comunidad}

En el centro de la Figura 1 se representa a la comunidad que es, a fin de cuentas, la receptora de las acciones y políticas; pero también los individuos, las familias y las comunidades pueden tomar acciones que favorezcan u obstaculicen el control y la prevención de enfermedades transmitidas por el vector 15 El rol de las familias en la implementación de acciones puede influir en la mejora o no de resultados en salud 20. En esta dimensión se agruparon factores relacionados con la participación de la comunidad en las acciones de prevención y la respuesta a las políticas orientadas a los individuos, familias, barrios y comunidades. En esta categoría se reconoce que la educación y distribución de información en salud influye en control del vector, pero que además que siempre las acciones están mediadas por las condiciones concretas de vida y de relaciones sociales de los grupos poblacionales expuestos.

La mayoría de los entrevistados resaltan la necesidad de trabajar con la comunidad y del trabajo intersectorial. Se relataron ejemplos sobre cómo se lograron mejorar los índices aédicos, mediante un trabajo de educación y la sensibilización sobre el problema, y sobre la responsabilidad de tomar acciones en su propio ámbito o vivienda.

Los entrevistados resaltan que en muchas comunidades no existe una percepción de riesgo de la enfermedad del dengue, al tratarse de un problema endémico con el que han convivido siempre. Por ello, la aparición del Zika y chikungunya ha generado el escenario favorable para lograr la sensibilización de la población y que se puedan tomar medidas.

Algunos entrevistados coinciden en que las poblaciones no poseen información correcta sobre el riesgo o sobre la importancia de tomar medidas en los hogares o lugares de trabajo. Muchas veces la información que brindan los medios de comunicación masivos o que circula en las redes sociales no es útil o aplicable; e incluso no es correcta, si se trata de noticias periodísticas aisladas que no provienen de campañas planificadas por expertos.

Se menciona que, a pesar de la implementación de programas educativos adecuados en algunas provincias o localidades, no se logra la sensibilización y, en consecuencia, el cambio esperado y sostenido en el tiempo, incluso en localidades donde ha habido brotes.

La implementación de algunas acciones ha tenido resistencias o no han sido aceptadas por lo población. Algunos entrevistados sostienen que, aunque la gente tiene conocimiento de las intervenciones preventivas, se ha fracasado en lograr la adherencia a las mismas, no las adoptan porque no se adaptan a sus hábitos y costumbres. Se mencionan algunas estrategias que generan rechazo en la población como el mal olor del repelente, las dificultades de usar ropa que cubra todo el cuerpo en zonas donde hace mucho calor, o las limitaciones económicas, por ejemplo, para la instalación de mosquiteros en el hogar, compra de ropa adecuada o repelentes.

A un nivel más estructural se menciona que otra barrera es trabajar con población de bajos recursos. La población que no tiene satisfecha sus necesidades básicas poco puede preocuparse por la prevención o no cuenta con los recursos para implementar las recomendaciones que reciban.

\section{- Opinión pública}

Entre la comunidad y el sistema de salud y otros sectores se ubica la opinión pública: como una dimensión transversal de gran peso. Esta dimensión considera la manera de pensar o el juicio común a la mayoría de las personas acerca de un asunto. Está formada por la tradición cultural y estimulada por los medios de comunicación. Esta categoría tiene un impacto en las decisiones políticas, la sensibilización y la implementación de intervenciones.

En esta dimensión nos referimos a la información que circula en los medios de comunicación masivos, en redes sociales o en redes más informales que de alguna u otra forma impacta en la opinión de la población. Las decisiones políticas están en gran medida influenciadas por las demandas de la población o por la presión que ejercen los medios de comunicación. La sociedad y los decisores reaccionan ante casos reportados en los medios de comunicación, a veces sobredimensionando la información real, y creando un pánico innecesario en la población. Por otro parte, sea por el periodismo 
o por los mensajes que circula por las redes sociales, se llega a transmitir información que de alguna forma no puede ser controlada por los ministerios. La desventaja que tiene esto es que puede circular información imprecisa o engañosa.

\section{Discusión}

En el abordaje cualitativo que se presenta aquí, expertos en el control del Ae. aegypti aportaron su conocimiento y experiencia acerca de la implementación de programas nacionales o regionales, ya sea como responsables, ejecutores, técnicos o investigadores. Del análisis se identificaron barreras percibidas en distintas dimensiones, sobre las cuales se debería intentar operar para maximizar las probabilidades de éxito de las intervenciones. Respecto a la dimensión de ambiente, surgen como barreras el cambio climático y la impredecibilidad de la aparición del vector. En la dimensión del sistema de salud, se observan como barreras la falta de recursos materiales y de personal capacitado, la priorización de temas de salud diferentes al control vectorial, la discontinuidad de los programas de control y la omisión de acciones preventivas, lo que es consistente con una publicación de Pérez et al. 21. En la dimensión del funcionamiento del estado o gobernanza, aparecen dificultades como la desarticulación entre los gobiernos locales y el gobierno central, la falta de integración del sector salud con otros sectores (ministerios de ambiente, educación, economía, etc.), y la falta de continuidad de los grupos técnicos responsables de los programas de control. En la dimensión de la opinión pública, se identifican como problemas la presión de los medios sobre las políticas públicas (como podría ser, la fumigación masiva) o la información incorrecta. En cuanto a la dimensión de la comunidad, es decir, las acciones que puede tomar la población en los ambientes cotidianos, conspiran contra una estrategia de control efectiva la falta de información o sensibilización sobre el riesgo, la pobreza y el rechazo o resistencia a adherir a las intervenciones, tal como lo reportan también otros estudios 9,22.

De todo lo anterior, se desprenden varias recomendaciones. Los programas de salud deben tomarse como políticas de estado, a fin de conseguir la continuidad y sostenibilidad de los mismos, independientemente de los cambios de gobierno, a fin de dar continuidad a los equipos técnicos a cargo. Otros estudios también revelaron la percepción de acciones insuficientes por parte del gobierno o descoordinación de las mismas 9,23,24,25. Se deben integrar las acciones entre distintos sectores (de salud y otros ministerios) y distintos niveles de gobernabilidad (nivel nacional, regional y local).

Este como otros estudios identifica la necesidad de educar y concientizar a la comunidad, a fin de conseguir una participación activa que no sólo se dé cuando haya brote de casos 9 . El desafío se presenta en cómo lograrlo, ya que muchas estrategias han fracasado, según reportan los expertos entrevistados, muchas campañas planificadas desde las esferas nacionales o regionales no contemplan las realidades locales. Es necesario realizar investigación sobre las necesidades de la población para adaptar los mensajes a los hábitos y costumbres, basar las estrategias de abordaje a la comunidad en marcos teóricos de cambio de comportamiento. También es necesario evaluar el impacto de estas acciones para tener más evidencia sobre estrategias efectivas.

El control vectorial constituye una de las principales medidas de prevención, aunque lograr ese objetivo representa un desafío para los países en desarrollo. El estudio que se presenta en este manuscrito forma parte de una investigación integral para abordar la complejidad de este problema sanitario. Los resultados de la investigación que aquí se presenta pueden ser leídos en conjunto con otros dos abordajes que lo complementan: una revisión sistemática con metaanálisis, que buscó identificar la evidencia científica, que respalda la implementación de las distintas intervenciones destinadas a controlar la densidad del vector 9, y un diálogo de decisores (policy dialogue) con una modalidad de taller presencial de priorización de intervenciones para el control del mosquito Ae. aegypti y de líneas de investigación 10 .

La principal fortaleza de este estudio cualitativo yace en que es el primer estudio que incluye la perspectiva de expertos temáticos en el control de Ae. aegypti de América Latina y Caribe, específicamente informado por los resultados de la revisión sistemática ${ }^{9}$ y plasmado en un diálogo de políticas 10 . Esta metodología deliberativa y colaborativa es una forma eficiente de triangular técnicas y estrategias de investigación para llegar a resultados más sólidos y consensos más amplios entre los actores relevantes involucrados en la solución de estos problemas de salud pública. 
Como limitaciones de este estudio, podría remarcarse el tamaño de muestra y el desequilibrio de participantes, que predominó para uno de los países, sin embargo, se debe resaltar que los hallazgos por país fueron consistentes con diferencias propias de las características locales. Sin embargo, este estudio no pretendió marcar las diferencias entre países. Las barreras y facilitadores mencionadas fueron similares en los países, en ese manuscrito se mencionan todas las barreras halladas.

\section{Conclusión}

Este estudio propone un marco de dimensiones de análisis construida desde los hallazgos; describe factores influyentes en la implementación de políticas que pueden orientar las futuras acciones desde un enfoque integrado en la región.

\section{Colaboradores}

Cada uno de los autores contribuyó sustancialmente en la producción del artículo.

\section{Informaciones adicionales}

ORCID: Elena Tapia-López (0000-0003-38926776); Ariel Bardach (0000-0003-4437-0073); Agustín Ciapponi (0000-0001-5142-6122); Andrea Alcaraz (0000-0002-4260-8239); Herney Andrés García-Perdomo (0000-0001-6945-8261); Silvina Ruvinsky (0000-0001-8729-1119); María Belizán (0000-0002-8739-5249).

\section{Agradecimientos}

Al bibliotecario Daniel Comandé por su asistencia en la obtención de manuscritos. A la Fundación Bunge y Born por el subsidio de investigación.

\section{Referencias}

1. Pan American Health Organization. Key messages for individuals and families regarding surveillance and control of Aedes aegypti: transmitter of dengue, chikungunya, Zika and other arbovirus diseases in the Americas. Washington DC: Pan American Health Organization; 2016.

2. Organización Panamericana de la Salud. Guía para la vigilancia de la enfermedad por el virus del Zika y sus complicaciones. Washington DC: Organización Panamericana de la Salud; 2016.

3. Wilder-Smith A, Chen LH, Massad E, Wilson ME. Threat of dengue to blood safety in dengue-endemic countries. Emerg Infect Dis 2009; 15:8-11.

4. Gubler DJ. Epidemic dengue/dengue hemorrhagic fever as a public health, social and economic problem in the 21 st century. Trends Microbiol 2002; 10:100-3. 
5. Toan NT, Rossi S, Prisco G, Nante N, Vivian $\mathrm{S}$. Dengue epidemiology in selected endemic countries: factors influencing expansion factors as estimates of underreporting. Trop Med Int Health 2015; 20:840-63.

6. Chang MS, Christophel EM, Gopinath D, Abdur RM. Challenges and future perspective for dengue vector control in the Western Pacific Region. Western Pac Surveill Response J 2011; 2:9-16.

7. França LS, Macedo CMA, Vieira SNS, Santos AT, Sanches GJC, Silva JM, et al. Challenges for the control and prevention of the Aedes aegypti mosquito. Rev Enferm UFPE on line 2017; 11:4913-8.

8. Organización Mundial de la Salud. Lucha contra el dengue. Estrategias de lucha antivectorial. http://www.who.int/denguecontrol/control_ strategies/es/ (accedido el Oct/2017).

9. Bardach AE, García-Perdomo HA, Alcaraz A, López ET, Gándara RAR, Ruvinsky S, et al. Interventions for the control of Aedes aegypti in Latin America and the Caribbean: systematic review and meta-analysis. Trop Med Int Health Dis 2019; 10.1111/tmi.13217.

10. Ciapponi A, Bardach A, Alcaraz A. Taller de priorización de intervenciones para el control del mosquito Aedes aegypti en América Latina y el Caribe: diálogo de políticas (policy dialogue). https://www.iecs.org.ar/mosquito/ (accedido el Oct/2018).

11. Glaser B. Discovery of grounded theory: strategies for qualitative research. London: Routledge; 2017.

12. Mays N, Pope C. Rigour and qualitative research. BMJ 1995; 311:109.

13. Tong A, Sainsbury P, Craig J. Consolidated criteria for reporting qualitative research (COREQ): a 32-item checklist for interviews and focus groups. Int J Qual Health Care 2007; 19:349-57.

14. O’Brien BC, Harris IB, Beckman TJ, Reed DA, Cook DA. Standards for reporting qualitative research: a synthesis of recommendations. Acad Med 2014; 89:1245-51.

15. Ghaffar A, Collins T, Matlin S, Olifson S. The 3D Combined Approach Matrix: an improved tool for setting priorities in research for health. Geneva: Global Forum for Health Research; 2009.
16. Ibarra AMS, Luzadis VA, Cordova MJB, Silva M, Ordoñez T, Ayala EB, et al. A social-ecological analysis of community perceptions of dengue fever and Aedes aegypti in Machala, Ecuador. BMC Public Health 2014; 14:1135.

17. Kaufmann D, Kraay A, Mastruzzi M. Governance matters VII: aggregate and individual governance indicators 1996-2007. Washington DC: World Bank; 2008.

18. Hufty M. Gobernanza en salud pública: hacia un marco analítico. Rev Salud Pública 2010; 12:39-61.

19. Hufty M, Báscolo E, Bazzani R. Gobernanza en salud: un aporte conceptual y analítico para la investigación. Cad Saúde Pública 2006; 22 Suppl:S35-45.

20. McDaniel SH, Campbell TL, Hepworth J, Lorenz A. Family-oriented primary care. Berlin: Springer Science \& Business Media; 2005.

21. Perez D, Lefevre P, Castro M, Toledo ME, Zamora G, Bonet M, et al. Diffusion of community empowerment strategies for Aedes aegypti control in Cuba: a muddling through experience. Soc Sci Med 2013; 84:44-52.

22. Elsinga J, van der Veen HT, Gerstenbluth I, Burgerhof JGM, Dijkstra A, Grobusch MP, et al. Community participation in mosquito breeding site control: an interdisciplinary mixed methods study in Curaçao. Parasit Vectors 2017; 10:434.

23. Flynn A. A study exploring the knowledge, attitudes and practices of young people regarding dengue fever and the extent of community involvement in vector control of the disease in Trinidad and Tobago. West Indian Med J 2012; 61:615-8.

24. Mirabal Jean-Claude M, Noriega Bravo V. Criterios del personal de apoyo en el trabajo contra el Aedes aegypti en Ciudad de La Habana 2006. Rev Cuba Salud Pública 2008; 34:0-0.

25. Sánchez L, Pérez D, Alfonso L, Castro M, Sánchez LM, Van der Stuyft P, et al. Estrategia de educación popular para promover la participación comunitaria en la prevención del dengue en Cuba. Rev Panam Salud Pública 2008; 24:61-9. 


\section{Abstract}

This study sought to describe experiences, barriers and facilitators to the implementation of interventions for controlling Aedes aegypti in Latin America and the Caribbean. A qualitative approach with semi-structured in-depth interviews with program implementation experts (19 participants in nine countries) was carried out. We used a software to codify the data and created matrices to compare them. Based on our findings, we developed a graphic representation of the theoretical dimensions that encompass the barriers and facilitators to the implementation of interventions. At the global level, the natural and built environment contributed to the mosquito's reproduction. In the health system, the lack of priority given to the problem and the scarcity of material and human resources are the most important obstacles. Sectors other than health must take responsibility for actions directed at improving social determinants of health. There are transversal barriers related to governance, lack of coordination between central and local levels, lack of continuity in terms of interventions and technical groups. The community faces problems such as lack of information, poverty or resistance to recommendations. Public opinion can have a positive or negative impact, indirectly influencing political decisions. This study proposes a framework of analytical dimensions based on our findings; describes factors that influence policy implementation, which can guide future actions from an integrated perspective.

Vector Control; Aedes; Qualitative Analysis

\section{Resumo}

O objetivo foi descrever experiências, barreiras e facilitadores na implementação de intervenções de controle do Aedes aegypti na América Latina e no Caribe. Uma abordagem qualitativa foi realizada com entrevistas semiestruturadas em profundidade com especialistas na implementação do programa (19 participantes de nove países). Foi usado um software para codificar os dados e foram feitas matrizes para comparação. Com base nos resultados, desenvolvemos uma representação gráfica das dimensões teóricas que agruparam as barreiras e facilitadores para a implementação das intervenções. Em nivel global, o ambiente natural e construído contribui para a reprodução do mosquito. No sistema de saúde, a falta de priorização do problema e a escassez de recursos materiais e humanos representam os obstáculos mais importantes. É necessário que outros setores, além do setor saúde, assumam ações de melhoria dos determinantes sociais da saúde. Existem barreiras transversais relacionadas à governança, como a falta de coordenação entre os níveis central e local e a falta de continuidade das intervenções e dos grupos técnicos. A comunidade enfrenta problemas como falta de informação, pobreza ou resistência a recomendações. A opinião pública pode ter um impacto positivo ou negativo, influenciando indiretamente as decisões políticas. Este estudo propõe um quadro de dimensões de análise construído a partir dos resultados; descreve fatores influentes na implementação de políticas que podem orientar ações futuras a partir de uma abordagem integrada.

Controle de Vetores; Aedes; Análise Qualitativa
Recibido el 10/May/2018

Versión final presentada el 17/Oct/2018

Aprobado el 23/Nov/2018 